\title{
Nucleotide sequence variation in mitochondrial COI gene among 147 silkworm (Bombyx mori) strains from Japanese, Chinese, European and moltinism classes
}

\author{
Kenji Yukuhiro ${ }^{1 *}$, Hideki Sezutsu', Toshiki Tamura ${ }^{2}$, \\ Eiichi Kosegawa ${ }^{3}$ and Makoto Kiuchi ${ }^{2}$ \\ ${ }^{1}$ Transgenic Silkworm Research Unit, Genetically Modified Organism Research Center, \\ National Institute of Agrobiological Sciences, Tsukuba, Ibaraki 305-8634, Japan \\ ${ }^{2}$ Division of Insect Sciences, National Institute of Agrobiological Sciences, Tsukuba, Ibaraki 305-8634, Japan \\ ${ }^{3}$ Genetic Resource Conservation Research Unit, Genetic Resource Center, National Institute of \\ Agrobiological Sciences, Hokuto, Yamanashi 408-0044, Japan
}

(Received 23 August 2011, accepted 1 December 2011)

\begin{abstract}
We characterized the nucleotide sequences of PCR-amplified mitochondrial COI fragments of 147 silkworm (Bombyx mori) strains that have been maintained in the National Institute of Agrobiological Sciences. Coding sequences (714 bp) of the 147 COI fragments were classified into eight haplotypes based on nucleotide differences at eight segregating sites. No length variation was identified in this region. The 5'-noncoding region showed different features, wherein changes in the number of $\mathrm{Ts}$ in the T-stretch, together with two base substitutions, were observed. As a result, the $147 \mathrm{COI}$ noncoding sequences were classified into six haplotypes. Combining the coding and noncoding regions, we identified 14 haplotypes. One of the 14 haplotypes, Hap1A was exclusively abundant in the Japanese native strain class, while this haplotype was less frequent in the other three native strain classes. This finding suggests that the Japanese strain class underwent significant genetic differentiation from the Chinese, European, and moltinism classes, when the each class is regarded as a population. Comparison of the nucleotide sequences to those of $B$. mandarina (which inhabits Japan) revealed changes that are significantly larger than those within either $B$. mori or B. mandarina. Furthermore, we detected no common haplotypes between them, which suggests the concept of suppressed gene flow between the two species.
\end{abstract}

Key words: Bombyx mori, Bombyx mandarina, COI haplotype, gene flow

\section{INTRODUCTION}

The silkworm Bombyx mori is a highly domesticated insect that has been artificially selected to increase silk production and facilitate rearing. Consequently, B. mori lacks the ability to find and approach its food source, mulberry leaves, during the larvae stage, and to fly in the adult stage. Commercial strains that have been bred to produce a large amount of silk also tend to have difficulty in mating. Such features prompt us to recognize that $B$. mori cannot live without human care; that is, it cannot survive in the field.

The domestication of $B$. mori is believed to have

Edited by Fumio Tajima

* Corresponding author. E-mail: kygnis@affrc.go.jp

Abbreviations: COI, cytochrome c oxidase subunit I; CDR, coding region; NCDR, noncoding region. occurred in China more than 5,000 years ago. The ancestor of $B$. mori is thought to be the wild mulberry silkworm B. mandarina, which inhabits China. These two species share common features such as carrying 28 chromosomes per haploid genome (Astaurov et al., 1959). Recent molecular phylogenetic studies support this hypothesis (Arunkumar et al., 2006).

During the domestication process, many silkworm strains have been yielded. They have differences in physiological traits, such as moltinism and voltinism, as well as phenotypic characteristics, such as differences in cocoon color, egg color, and body size. Such remarkable differences suggest that significant levels of genetic variation exist in $B$. mori genetic resources.

Following completion of the silkworm genome project (International Silkworm Genome Consortium, 2008), the genetic variation in $B$. mori has been carefully observed. 
Chinese research groups demonstrated significant levels of variation between the nucleotide sequence of nuclear and mitochondrial genomes (Xia et al., 2009; Li et al., 2010, respectively). However, their results have not effectively contributed to understanding the population structure in the whole silkworm genetic resource. Because of the small size of samples, their results were not always representatives of whole silkworm genetic resource. In addition, they used quite small number of $B$. mandarina samples derived from limited area of China.

The National Institute of Agrobiological Sciences (NIAS) Japan has maintained hundreds of geographic strains of $B$. mori as genetic resources. These geographic strains are preserved as a collection of native and improved strains (Sorita, 1991; Kosegawa et al., 1997). The four native strain classes (Japanese, Chinese, European, and tropical) were derived from many local areas. In addition, the moltinism strain class, which includes local strains characterized by moltinism mutations, which differ from the standard tetramolter, has traditionally been maintained as the native strain.

To demonstrate the level of genetic variation that has been maintained in the $B$. mori genetic resource at the nucleotide level, we conducted DNA sequencing and subsequent DNA polymorphism analysis of partial mitochondrial COI gene fragments mediated by PCR amplification. The COI gene is a major target gene of the DNA Barcoding Project (Kress et al., 2005). DNA barcoding is a method to identify species of organisms based on matching to short genetic markers. Therefore, a series of sequence results are highly applicable to phylogenetic analysis of the Bombycidae species, particularly the probable ancestor of the silkmoth, B. mandarina.

We characterized mitochondrial COI nucleotide sequences from 147 native silkworm strains and identified eight segregating nucleotide sites, wherein two different nucleotides were observed in the COI-coding region. Furthermore, length variation, which was caused by variable numbers of thymine $(\mathrm{T})$ stretches, were observed in the 5'-noncoding region. In total, we detected 14 different haplotypes. The 14 COI haplotypes differentially appeared in the four native strain classes, which is indicative of significant genetic differentiation in the Japanese strain class, as compared to others.

We further discuss the relationship between $B$. mori and Japanese $B$. mandarina, which are likely separated by more than a million years from a common ancestor of B. mori (Yukuhiro et al., 2002; Pan et al., 2008).

\section{MATERIALS AND METHODS}

Genomic DNA samples of native silkworm strains The DNA samples used in this study were similar to those in Tomita et al. (1997), except we excluded the tropical strains because of the small number of strains kept in NIAS. We analyzed 48 Japanese, 50 Chinese, 28 European, and 21 moltinism strains. The European strain samples consisted of 26 native and two improved strains, while other three strain classes exclusively contained native strains. The list of strains used in this study is presented in Table 1 . The purification method of genomic DNAs was described in Tomita et al. (1997).

PCR amplification of target DNA fragments We used a pair of COI-specific primers TY-J-1460 and Cl-N2191 (Simon et al., 1994). Nucleotide sequences of the two primers are as follows:

TY-J-1460: 5'-TACAATTTATCGCCTAAACTTCAGCC-3' Cl-N-2191: 5'-CCCGGTAAAATTAAAATATAAACTTC-3'

To read both terminus of amplified fragment, we linked the T7 and M13 reverse primer sequences to TYJ-1460 and Cl-N-2191 primers, respectively, at the 5' terminus.

A reaction mixture contained $1 \times$ Ex Taq buffer (TaKaRa, Kyoto, Japan), $0.8 \mathrm{mM}$ dNTP, $0.2 \mu \mathrm{M}$ each primer, 0.5 unit of Ex Taq (TaKaRa), and approximately $5 \mathrm{ng}$ of genomic DNA, which contained some amount of mtDNA, in a $20 \mu \mathrm{l}$ reaction mixture. Cycling conditions were as follows: 2 -min incubation at $96^{\circ} \mathrm{C}$ and 35 cycles of $15 \mathrm{~s}$ at $96^{\circ} \mathrm{C}, 15 \mathrm{~s}$ at $40^{\circ} \mathrm{C}$, and $1 \mathrm{~min}$ at $68^{\circ} \mathrm{C}$, followed by a 5 -min incubation at $68^{\circ} \mathrm{C}$. We confirmed amplification by agarose gel electrophoresis followed by detection under Gel Red (Biotium, CA, USA) staining.

DNA sequencing Prior to DNA sequencing, we removed the remaining primers and dNTPs by treatment with ExoSap-IT (USB Corp., Cleveland, OH, USA) according to manufacturer's protocol. We used the ABI BigDye Terminator ver. 3.1 (Applied Biosystems, Foster City, CA, USA) for DNA sequencing. The sequencing mixture (10 $\mu \mathrm{l})$ contained the following components: $1.5 \mu \mathrm{l} 5 \times$ buffer (Applied Biosystems), $1 \mu \mathrm{l}$ Premix (Applied Biosystems), $0.1 \mu \mathrm{l}$ of $20 \mu \mathrm{M}$ primer for sequencing, $5.4 \mu \mathrm{l}$ of $\mathrm{dH}_{2} \mathrm{O}$, and $2.0 \mu \mathrm{l}$ of PCR purified product. For the initial sequencing, we used M13 reverse primers. Cycling conditions were as follows: 2 -min incubation at $96^{\circ} \mathrm{C}$ followed by 35 cycles at $96^{\circ} \mathrm{C}$ for $15 \mathrm{~s}, 50^{\circ} \mathrm{C}$ for $10 \mathrm{~s}$, and $60^{\circ} \mathrm{C}$ for 4 min. Upon PCR completion, we purified the sequencing products on a Sephadex column. We used the ABI Prism 3730 (Applied Biosystems) to detect sequencing signals.

When a read did not reach the 5' terminus of the COI fragment, we sequenced it using a T7 primer to read the opposite strand. We also used three additional primers (COI_443_2U, COI_221L, and COI_576L) for sequencing. Nucleotide sequences of the three primers were as follows: COI_443_2U: 5'-TATTGCACACAGAGGAAGATCTG-3', COI_221L: 5'-GGRKTWCCTAATTCTGCTCGA-3', and COI_576L: 5'-AATGCTGTAATTCCTACAG-3'. 
Table 1. List of silkworm strains used in this study

\begin{tabular}{|c|c|c|c|}
\hline Japanese strains & Chinese strains & European strains & Moltinism strains \\
\hline Akajuku & Amoi moricaud & Yellow cocoon of Asia & Chojo \\
\hline Akako & Benishina & Ascoli & Choyo \\
\hline Aojuku (A) & Segregated white & Yellow cocoon of Ascoli & European No.7 trimolter \\
\hline Aojuku (Ichinomiya) & Chahan & Bagdad & Trimolter of Kan \\
\hline Agunisan & C spherical cocoon & 91 Bagdad & Trimolter of Korai \\
\hline Chikuma & Daiento & Pione & Menyo \\
\hline Chusuotsu & Hekiren & Blanc des C'evennes & Sainan \\
\hline Dainyorai & Hiko & Ginto & Sanko \\
\hline Datenishiki & Kaijo & Gubbio & Trimolting white \\
\hline Himeko & Kanko & Golden pink & Trimolter of Santo \\
\hline Hinode (Odaka) & Kankoshaken & Black moth & Sen No.3 \\
\hline Kakumata (Aramomi) & Kansen & MaiellaZebra & Golden yellow cocoon of Shisen \\
\hline Kokin & Golden yellow & Yellow cocoon of Maruke & Trimoter of Shisen \\
\hline Kinkoshu & Knobbed & No. 500 & Kyokuko \\
\hline Kinshoku (Maebashi) & Kohoku & No. 65 & OK39 \\
\hline Koishimaru & Kuroko (Shimane) & Rosa & $\mathrm{VC} 1$ \\
\hline (Koishimaru Watari) & Kyugyoku & S No. 9 & Chogosan No. 1 \\
\hline Kuniichi & Nanko & Spain & Pentamolting white \\
\hline Matamukashi & Oha & St. Julien & Pentamolting black \\
\hline Murasakiko & Renshin & Szegsard & Pentamolter of Kyushu Univ. \\
\hline Onichijimi & Seikyo & Yellow cocoon of Turkey & $\mathrm{N}-\mathrm{S}$ \\
\hline Ryukyu multi-pupal cocoon (Kiaya) & Sekko & Var & \\
\hline Ryukyu multi-pupal cocoon (Shizuoka) & Shakugen & Y4 & \\
\hline Sagami & Chinese white cocoon & y35 & \\
\hline Sakurahime & Shinshocho & y39 & \\
\hline Seihaku (Shizuoka) & Shokei & Yuhaku & \\
\hline Sekaiichi & Shuko & European No. 16 (Old) & \\
\hline Tadamiko & $\mathrm{S}$ & European No. 16 (New) & \\
\hline Tenryuseihaku & Tenmon & & \\
\hline Wako & Tokuishin & & \\
\hline Aojuku (Hanyuda) & Tsunoshina & & \\
\hline Chiyozuru & Uryu & & \\
\hline Chusu & Y10 & & \\
\hline Kinshoku (Matsumoto) & Yoko & & \\
\hline Kojiki & Daiankyo & & \\
\hline Kunitomi & Daikojo & & \\
\hline Kuroko (Takahashi) & Daizo (Nagano) & & \\
\hline Kuroko & Daizo (Matsumura) & & \\
\hline Nihonichi & Hakuyoen & & \\
\hline Daiaojuku & Kinkomaru & & \\
\hline Okusa & Kosetsu & & \\
\hline Onishiki & Striped & & \\
\hline Seihaku (Yamagata) & Shoka & & \\
\hline Shohaku (Maebashi) & Shoko & & \\
\hline Shohaku (Taketoyo) & $208 \mathrm{AB}$ & & \\
\hline Tamanashi kasuri & $209 \mathrm{AB}$ & & \\
\hline Tanegashima & Low 209 & & \\
\hline \multirow[t]{4}{*}{ Tsunomata (Shizuoka) } & 210 & & \\
\hline & 213 & & \\
\hline & 214 & & \\
\hline & 216 & & \\
\hline
\end{tabular}

Sequence analysis We edited the nucleotide sequences using ATGC (Genetyx Co., Tokyo, Japan) followed by DNA sequence alignment using Clustal W (Thompson et al., 1997) in MEGA (Tamura et al., 2007; Kumar et al., 2008). After aligning the nucleotide sequences, we prepared a fasta-formatted sequence data set. We analyzed this data set using DnaSP version 5.0 (Librado and Rozas, 2009) to detect segregating sites and to identify constitu- tive haplotypes. We further used this software to estimate the population genetic parameters, $S, \pi$, and $\mathrm{F}_{\mathrm{st}}$. TCS 1.21 (Clement et al., 2000) was used to construct the maximum-parsimony haplotype network.

Fourteen DNA sequences of $B$. mori COI haplotypes reported here are available in the DDBJ/EMBL/GenBank databases under the accession numbers AB649182, AB649183, AB649184, AB649185, AB649186, AB649187, 
AB649188, AB649189, AB649190, AB649191, A1DB649192, AB64918493, AB649194, and AB649195 for BmoHap1A, BmoHap1B, BmoHap1D, BmoHap2A, BmoHap2C, BmoHap2D, BmoHap2E, BmoHap2F, BmoHap3A, BmoHap4D, BmoHap5D, BmoHap6D, BmoHap7A, and BmoHap8D, respectively.

\section{RESULTS AND DISCUSSION}

We successfully amplified COI fragments from 147 preserved silkworm strains. The COI fragment can be divided into two regions; the 5'-noncoding region (NCDR) and coding region (CDR). Initially, we separately analyzed the two regions because they showed different features in sequence variation.

Segregation sites in the COI coding region We identified eight segregating sites wherein two different nucleotides were observed at 714 CDR nucleotide sites (Fig. 1A). In the eight segregating sites, we detected eight transitions (T vs. C: 4; A vs. G: 4) and no transversions. Three of eight base changes were nonsynonymous (Fig. 1A). At the $41^{\text {st }} \mathrm{CDR}$ site, a $\mathrm{C}$ to $\mathrm{T}$ transition caused a Thr to Met mutation. G to A transition at site 238 induced a Val to Ile replacement, while a G to A transition at site 256 yielded an Ala to Thr nonsynonymous change. We did not identify any indels in the CDR. This is indicative of a non-length polymorphism in the COI CDR, showing significant functional constraint. To assess the magnitude of genetic variation in silkworm COI coding sequences in the 147 preserved strains, we estimated nucleotide diversity as $\pi$. The estimated $\pi$ score was calculated to be $0.00080 \pm 0.00008$. Estimated S (the number of segregating sites) and the $\pi$ scores in four types of the native strains are shown in Table 2 .

Haplotypes in the coding region Based on the differences in nucleotide sequence, we identified eight haplotypes. We tentatively termed the eight haplotypes for CDR Hap1 to Hap8 (Fig. 1A). A maximum-parsimony haplotype network generated by TCS 1.21 (Clement et al., 2000) is shown in Fig. 2A. The score of haplotype diversity (Hd) for the $147 \mathrm{COI}$ sequences was calculated to be $0.5001 \pm 0.0319$ (Table 2). Differences between the individual haplotypes were quite small, which is consistent with the low estimate of $\pi$.

The frequency of each haplotype is demonstrated in Table 3, clearly indicating biased occurrences of haplotypes. The most abundant haplotype was Hap2, whose frequency was $0.639(94 / 147)$. The next more frequent

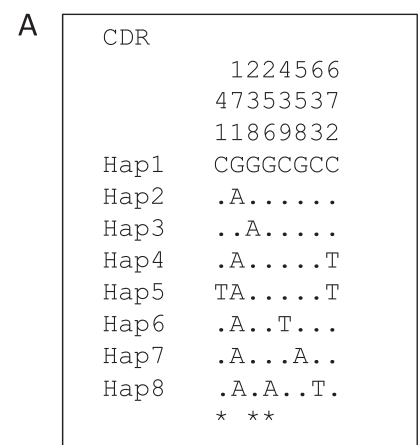

B

Whole NCDR

$$
\begin{aligned}
& 1234567890 \quad 1234567890 \quad 1234 \\
& \text { HapA ATTTTATTTT TTTT-CTTTT TTAG } \\
& \text { НарВ ................. } \\
& \text { Нарс } \ldots \ldots \ldots \ldots \ldots
\end{aligned}
$$

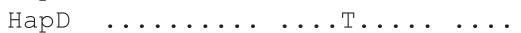

\begin{tabular}{|c|c|c|}
\hline $\begin{array}{l}\text { BmoHap1A } \\
\text { BmoHap1B } \\
\text { BmoHap1D } \\
\text { BmoHap2A } \\
\text { BmoHap2C } \\
\text { BmoHap2D } \\
\text { BmoHap2E } \\
\text { BmoHap2F } \\
\text { BmoHap3A } \\
\text { BmoHap4D } \\
\text { BmoHap5D } \\
\text { BmoHap6D } \\
\text { BmoHap7A } \\
\text { BmoHap8D }\end{array}$ & $\begin{array}{c}11111 \\
745678 \\
\mathrm{TT}-\mathrm{CTT} \\
\text { А. Т... } \\
\ldots \mathrm{T} \ldots \\
\ldots-\ldots \\
.--.-- \\
\ldots \mathrm{T} \ldots \\
.-. \ldots \\
\ldots-\mathrm{T} \ldots \\
\ldots-\ldots \\
\ldots \mathrm{T} \ldots \\
\ldots \mathrm{T} \ldots \\
\ldots \mathrm{T} \ldots \\
\ldots-\ldots \\
\ldots \mathrm{T} \ldots \\
\\
\text { NCDR }\end{array}$ & 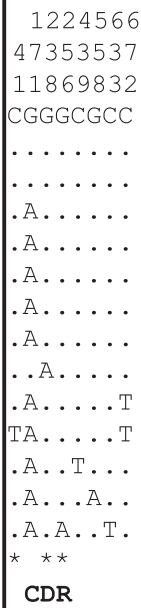 \\
\hline
\end{tabular}

$$
\begin{aligned}
& \text { Наре } \quad \ldots \ldots \ldots \ldots \ldots \\
& \mathrm{HapF} \quad \ldots \ldots \ldots \ldots \ldots . \mathrm{T} \ldots \ldots \ldots
\end{aligned}
$$$$
\star \star \star \star * \star * \star * \star * \star * \star * \star * \star * *
$$

C

Fig. 1. Segregating nucleotide sites in the B. mori COI nucleotide sequences. A. Segregating nucleotide sites in the B. mori COI coding region (CDR) and the eight haplotypes detected in the CDR. B. Aligned nucleotide sequences of six haplotypes identified in COI noncoding region (NCDR). Vertical numbers represent nucleotide positions in either the NCDR or CDR using the largest-sized flanking sequence from HapB as a standard. A hyphen means a deleted nucleotide. T-stretch region is marked by an*. We identified six haplotypes, A to F, in the NCDR. C. Segregating nucleotide sites in the B. mori COI coding region (CDR) combined with the 5'-noncoding region (NCDR). and 14 haplotypes identified. Vertical numbers represent nucleotide positions in the CDR, wherein a possible start site is defined as 1 . An * signifies a nonsynonymous nucleotide difference. A dot indicates an identical nucleotide. A hyphen means a deleted nucleotide. 
Table 2. Genetic variation scores of Bombyx mori native strains and 11 samples of Japanese B. mandarina in the COI coding region $(\mathrm{CDR})$

\begin{tabular}{lcccccc}
\hline \hline & $\begin{array}{c}\text { Number of } \\
\text { strains or } \\
\text { samples }\end{array}$ & $\begin{array}{c}\text { Total number } \\
\text { of nucleotide } \\
\text { sites }\end{array}$ & $\begin{array}{c}\text { Number of } \\
\text { segregating } \\
\text { sites (S) }\end{array}$ & $\begin{array}{c}\text { Number of } \\
\text { haplotypes }\end{array}$ & $\begin{array}{c}\text { Haplotype } \\
\text { diversity } \\
(\text { Hd) }\end{array}$ & $\begin{array}{c}\text { Nucleotide } \\
\text { diversity }(\pi)\end{array}$ \\
\hline B. mori (Total) & 147 & 714 & 8 & 8 & 0.5001 & 0.00080 \\
Japanese strains & 48 & 714 & 2 & 3 & 0.4140 & 0.00059 \\
Chinese strains & 50 & 714 & 4 & 5 & 0.2914 & 0.00048 \\
European strains & 28 & 714 & 1 & 2 & 0.2540 & 0.00036 \\
Moltinism strains & 21 & 714 & 5 & 5 & 0.4238 & 0.00079 \\
\hline B. mandarina* (Japan) & 11 & 714 & 6 & 7 & 0.8730 & 0.00224 \\
\hline
\end{tabular}

*Accession numbers of the sequences used in this survey are as follows: AF167266, AF167267, AF167268, AF167269, AF167270, AF167271, AF167272, AF167273, AF167274, AF167275, and AF167280.

Table 3. Haplotype classification of Bombyx mori native strains

\begin{tabular}{|c|c|c|c|c|c|c|c|c|c|c|c|c|c|c|}
\hline $\begin{array}{l}\text { Haplotypes } \\
\text { (Total) }\end{array}$ & $\begin{array}{c}\text { Bmo } \\
\text { Hap } \\
1 \mathrm{~A}\end{array}$ & $\begin{array}{c}\text { Bmo } \\
\text { Hap } \\
1 \mathrm{~B}\end{array}$ & $\begin{array}{c}\text { Bmo } \\
\text { Hap } \\
\text { 1D }\end{array}$ & $\begin{array}{c}\text { Bmo } \\
\text { Hap } \\
2 \mathrm{~A}\end{array}$ & $\begin{array}{c}\text { Bmo } \\
\text { Hap } \\
2 \mathrm{C}\end{array}$ & $\begin{array}{c}\text { Bmo } \\
\text { Hap } \\
\text { 2D }\end{array}$ & $\begin{array}{c}\text { Bmo } \\
\text { Hap } \\
2 \mathrm{E}\end{array}$ & $\begin{array}{c}\text { Bmo } \\
\text { Hap } \\
2 \mathrm{~F}\end{array}$ & $\begin{array}{c}\text { Bmo } \\
\text { Hap } \\
3 \mathrm{~A}\end{array}$ & $\begin{array}{c}\text { Bmo } \\
\text { Hap } \\
4 \mathrm{D}\end{array}$ & $\begin{array}{c}\text { Bmo } \\
\text { Hap } \\
5 \mathrm{D}\end{array}$ & $\begin{array}{l}\text { Bmo } \\
\text { Hap } \\
6 \text { D }\end{array}$ & $\begin{array}{c}\text { Bmo } \\
\text { Hap } \\
7 \mathrm{~A}\end{array}$ & $\begin{array}{c}\text { Bmo } \\
\text { Hap } \\
8 \mathrm{D}\end{array}$ \\
\hline Japanese strain class & 29 & 5 & 1 & 4 & 1 & 6 & 1 & 0 & 1 & 0 & 0 & 0 & 0 & 0 \\
\hline Chinese strain class & 4 & 0 & 0 & 10 & 0 & 31 & 0 & 1 & 0 & 2 & 1 & 1 & 0 & 0 \\
\hline European strain class & 3 & 0 & 1 & 18 & 0 & 6 & 0 & 0 & 0 & 0 & 0 & 0 & 0 & 0 \\
\hline Moltinism strain class & 2 & 0 & 0 & 9 & 0 & 7 & 0 & 0 & 0 & 1 & 0 & 0 & 1 & 1 \\
\hline Total & 38 & 5 & 2 & 41 & 1 & 50 & 1 & 1 & 1 & 3 & 1 & 1 & 1 & 1 \\
\hline
\end{tabular}

A

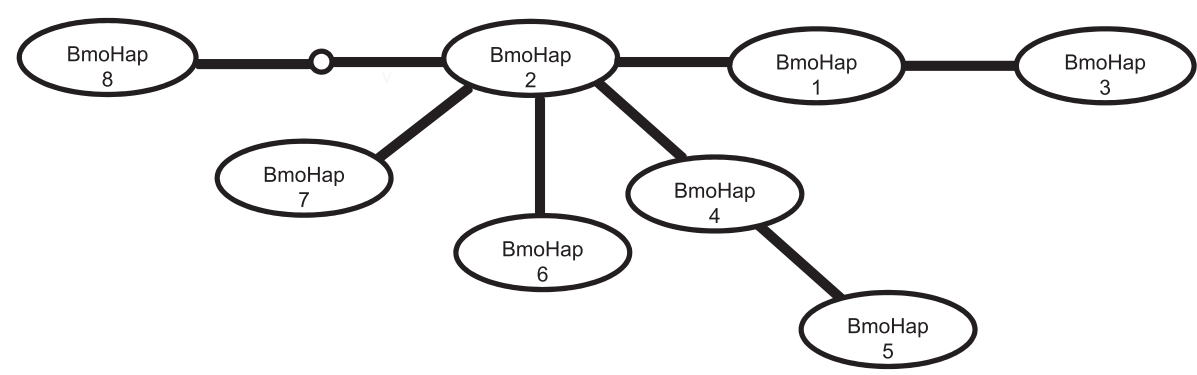

B

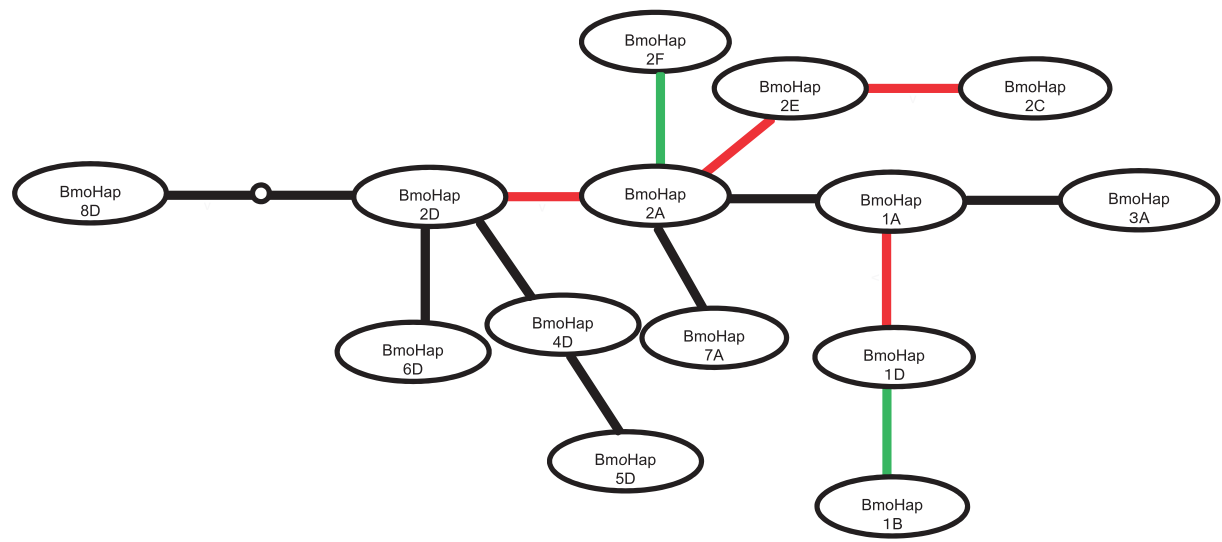

Fig. 2. Maximum-parsimony haplotype network of B. mori COI sequences. A. CDR. B. NCDR and CDR. A bar signifies a mutation. An open circle means an undetected haplotype. In Fig. $2 \mathrm{~B}$, a blue bar signifies a CDR base change, while a red bar indicates a change in the number of $\mathrm{T}$ in the NCDR. A green bar represents a base change in the NCDR. 
haplotype was Hap1 with a frequency of 0.306 (45/147). The other six haplotypes were less frequent; Hap4 appeared only three times, while the remaining five were singletons.

As shown in Fig. 1A, the nucleotide sequence of Hap1 differed from that Hap2 at site 171, where Hap1 had a G while Hap2 had an A. Hap3 shared an identical sequence to Hap1 except for site 238, where Hap3 carried an A and Hap1 had a G. As described above, this base change was nonsynonymous.

As shown in Fig. 2A, other haplotypes (Hap4, 5, 6, 7, and 8) were derived from Hap2. The nucleotide sequences of Hap4, 6, and 7 differed from that of Hap2 at sites 633, 439, and 538, respectively (Fig. 1A). An additional transition of $\mathrm{C}$ to $\mathrm{T}$ at site 41 on Hap4 yielded Hap5, and two extra transitions at sites 256 and 633 on Hap2 induced Hap8 (Fig. 1A). Note that Hap3, Hap5, and Hap8, which carried amino acid changes, were singletons.

Differential occurrences of haplotypes among the four native strain classes Heterologous distributions of the eight haplotypes, particularly Hap1 and Hap2, were clearly observed between the four native strain classes (Japanese, Chinese, European and moltinism). As shown in Table 3, the most frequent haplotype was Hap1 in the Japanese strains. In contrast, the other three native strains preferentially carried Hap2.

To assess the magnitude of variation at the nucleotide level within native strain classes, we estimated $S, \pi$, and haplotype diversity (Hd) for each of the four classes. Scores are summarized in Table 2. Despite the difference in the number of haplotypes between preserved strain classes, we did not observe remarkable differences in $\mathrm{S}, \pi$, and Hd scores. This finding infers that Japanese strain class underwent less strong bottleneck effect.

Although Tomita et al. (1997) reported higher levels of genetic variation in Chinese strain classes (compared to the others) by examining the abundance of mariner-like elements mediated by PCR-amplification, our approach did not result in clear differences among the four classes. This discrepancy likely arose from the relatively small number of variable sites in the present study. If a larger fragment were analyzed, clearer differences may be obtained.

To assess the differentiation level between native strain classes, we estimated a series of $\mathrm{F}_{\mathrm{st}}$ (fixation index). As shown in Table 4A, the results were highly biased; that is, $\mathrm{F}_{\mathrm{st}}$ scores between the Japanese strain and the others ranged from 0.4 to 0.5 , while those among three native strain classes (excluding Japanese) were quite low (approximately 0). These results clearly demonstrate genetic differentiation of the Japanese strain class from others.
Table 4. $\mathrm{F}_{\text {st }}$ scores

\begin{tabular}{lcrl}
\hline \hline A CDR & & & \\
\hline & & & \\
\cline { 2 - 4 } Chinese & 0.5366 & & \\
European & 0.5149 & 0.0012 & \\
Moltinism & 0.4601 & -0.0199 & -0.0164 \\
& & & \\
B NCDR + CDR & & & \\
\hline & & & \\
Chinese & 0.4863 & & \\
European & 0.4592 & 0.0012 & \\
Moltinism & 0.4218 & -0.0199 & -0.0164 \\
\hline
\end{tabular}

Haplotype classification of silkworm COI sequences including the noncoding region We analyzed the 5'noncoding region of the COI gene. Of the 147 sequences, we identified six different sequence types (HapA to F; Fig. 1B), wherein one transition between $\mathrm{C}$ and $\mathrm{T}$ (HapA vs. HapF), one transversion between $\mathrm{A}$ and $\mathrm{T}$ (HapA vs. HapB), and a difference in the number of thymine bases (T) in sites 7 to 15 were observed. The last event was associated with sequence slippage, such as a microsatellite sequence variation.

HapA was the most common at a frequency of 0.551 (81/ 147), followed by HapD (0.395:58/147). Although HapB appeared in five strains (0.034), the others were singletons.

As shown in Fig. 1C, tight linkage between coding and noncoding haplotypes was not observed. For example, Hap1 in the coding region was associated with HapA, HapB, and HapD, while Hap2 combined with all noncoding haplotypes, except for HapB. Hereafter, we analyzed a combined set of coding and noncoding sequences as a unit (Fig. 1C).

In Fig. 2B, we show a maximum-parsimony haplotype network of combined sequence sets. The differences in the parsimony networks between Fig. 2A and Fig. 2B were derived from a subdivision of Hap1 and Hap2. $\mathrm{F}_{\mathrm{st}}$ analysis also indicated heterogeneity of the Japanese strains from the others (Table 4B).

Overall, the Chinese, European, and moltinism strains shared a common feature, the mtCOI gene pool, but the Japanese strain possessed a different one. This suggests that the European and moltinism strain classes were derived from the Chinese strains without undergoing severe genetic drift. In contrast, the Japanese strains may have experienced biased sampling of COI haplotypes to change their population structure.

Genetic variation in nuclear gene of silkworm and comparison with that of $\mathrm{COI}$ We detected genetic differentiation in mitochondrial COI sequence of $B$. mori. When comparing the result with those of nuclear genes, we can use limited amount of information on genetic variation in nuclear genes. For example, Kim et 
al. (2008) analyzed nine independent intronic sequences from $25 \mathrm{~B}$. mori strains including four Japanese ones in order to develop genetic markers for use in strain identification. Despite use of only 25 strains, many haplotypes were detected in all of analyses. For example, 15 haplotypes were identified in cytoplasmic action A4 second intronic sequences, wherein 29 nucleotide changes and two events of indels were observed in 533 nucleotide sites. Note that 14 haplotypes were identified in 147 COI sequences of the present study. The excess number of haplotypes relative to that of COI is likely to come from less stringent functional constraint on intronic sequences. Moreover, two out of four Japanese originated strains were classified into a haplotype wherein Chinese, European and Korean strains were observed.

Recently, Yu et al. (2011) demonstrated that Tyrosine hydroxylase (TH) gene had extremely low amount of nucleotide diversity relative to Chinese $B$. mandarina. The TH gene was considered to be one of the target genes for silkworm domestication through multiple approaches of population genetic analyses. Note that despite of analyzing the gene under the artificial selection during domestication process and use of 15 strains, the 13 haplotypes based on nucleotide change was detected. Furthermore, Yu et al. (2011) demonstrated clear difference in haplotype structure between the gene under the artificial selection and neutral genes. Their result showed a common feature to that of Kim et al. (2008), that is, intronic sequences or sequences including introns are relatively abundant in haplotype to that of COI even in genes under the artificial selection.

\section{Similarity of silkworm COI sequences to Japanese} B. mandarina $B$. mori mates with $B$. mandarina (which inhabits Japan) to generate fertile progenies, although the number of chromosomes differs between them (B. mori: $\mathrm{n}=28$; Japanese $B$. mandarina: $\mathrm{n}=27$; Sasaki, 1898; Kawaguchi, 1928). The cross between a $B$. mori male and a $B$. mandarina female is almost impossible without human intervention because the former cannot approach the latter successfully even in capture. Successful mating only occurs between $B$. mori females and $B$. mandarina males. As mitochondrial DNA is maternally inherited, the hybrid progeny between $B$. mori females and $B$. mandarina males carry mitochondrial DNA from B. mori. If gene flow had continued to occur between $B$. mori and $B$. mandarina, we could detect mtDNA derived from $B$. mori in the $B$. mandarina natural population.

In this study, we detected eight haplotypes in COI CDR, six in COI NCDR and 14 in the combined data set of 147 B. mori preserved strains (Fig. 1, A-C). To confirm the occurrence of gene flow between $B$. mori and $B$. mandarina, we analyzed 11 COI DNA sequences of Japanese B. mandarina (available in NCBI). Addition- ally, we used a Korean B. mandarina COI sequence, as well as that of Chinese $B$. mandarina, for the analysis. The 11 Japanese $B$. mandarina COI sequences were classified into seven haplotypes based on seven segregating sites in the coding region (Fig. 3). Although four of the seven segregating sites were silent, three of seven segregating sites underwent nonsynonymous changes: a $\mathrm{C}$ to $\mathrm{T}$ change caused a Ser to Leu replacement at site 332 , a G to A change caused a Ser to Lys replacement at site 338, and a $\mathrm{G}$ to $\mathrm{A}$ transition caused a nonsynonymous change of Gly to Ser at site 409 . The $\pi$ value was estimated to be $0.00224 \pm 0.00046$ (Table 2).

Japanese $B$. mandarina sequences had a NCDR, whose organization was different from that of $B$. mori (The data of $B$. mori shown in Fig. 1B). This was caused by the addition of two AC dimers into the $\mathrm{T}$ stretch (data not shown). Because the difference in the repetitive structure between B. mori and Japanese B. mandarina gave us unreliable nucleotide sequence alignment results, we excluded the NCDR from this comparison.

The comparison of CDR nucleotide sequences clearly revealed that none of the seven Japanese $B$. mandarina COI haplotype sequences were identical to the eight $B$. mori sequences (Fig. 3). Eleven base substitutions were detected between $B$. mori and Japanese B. mandarina (Fig. 3). Figure 4 demonstrates the separation of Japanese $B$. mandarina and $B$. mori combined with Chinese and Korean B. mandarina. Note that the eleven base substitutions are synonymous. It means that $B$. mori and Japanese $B$. mandarina share an identical amino acid sequence for the COI fragments, strongly sug-

\begin{tabular}{|c|c|c|c|}
\hline [ & 112222333 & 3444444455 & $556666667]$ \\
\hline[ & 4573455233 & 9001238813 & $581356790]$ \\
\hline [ & 1618306528 & 9597393667 & $868346208]$ \\
\hline \#BmoHap1 & CTGGTCGTCG & СTGCTCTTTC & GCACTACTA \\
\hline \#BmoHap2 & $\ldots$ A..... & $\ldots \ldots \ldots$ & $\ldots \ldots \ldots$ \\
\hline \#BmoHap 3 & $\ldots$..... & $\ldots \ldots \ldots$ & $\ldots \ldots \ldots$ \\
\hline \#BmoHap 4 & $\ldots$ A...... & $\ldots \ldots \ldots$ & $\ldots \ldots \mathrm{T} \ldots$ \\
\hline \#BmoHap 5 & T.A..... & $\ldots \ldots \ldots$ & $\ldots \ldots$ T. \\
\hline \#BmoHap 6 & $\ldots$ A...... & $\ldots$. . . & $\ldots \ldots \ldots$ \\
\hline \#BmoHap 7 & $\ldots A \ldots \ldots$ & $\ldots \ldots \ldots$ & A........ \\
\hline \#BmoHap 8 & .А...А. & $\ldots \ldots \ldots$ & $\ldots$ T... \\
\hline \#BmaC_Hap1 & .А. Т... & $\ldots \ldots \ldots$ & $\ldots$ G. . . . \\
\hline \#BmaK_Hap1 & $\ldots A \ldots \ldots$ & T....... & $A \ldots \ldots G$ \\
\hline \#BmaJ_1 & .CA.C..C.. & TС.тСтС.Ст & AT..CGT. \\
\hline \#BmaJ_2 & .CA.C....A & TC.TСТС.СТ & AT . CGT . \\
\hline \#BmaJ_3 &. CA.C.... & TCATCTC.CT & $\mathrm{AT} \ldots \mathrm{CGT} \ldots$ \\
\hline \#BmaJ_4 & $. C A . C \ldots$ & TC.TCTCCCT & AT..CGT. \\
\hline \#BmaJ_5 &. CA.C.... & TC.TCTC.CT & AT..CGT. \\
\hline \#BmaJ_6 & .CA.C..C.. & TC.TCTC.CT & AT..CGTC. \\
\hline \#BmaJ_7 & . CA.C..CT. & TC.TCTC.CT & AT..CGT. \\
\hline
\end{tabular}

Fig. 3. Variable nucleotide sites in B. mori and the Chinese, Korean, and Japanese B. mandarina COI coding region. Vertical numbers represent nucleotide positions. Bmo: B. mori; BmaC, BmaK, and BmaJ: Chinese, Korean, and Japanese B. mandarina, respectively. A dot represents the same base as that of BmoHap1. Accession numbers of $\mathrm{BmaC}$ and $\mathrm{BmaK}$ sequences are AF167278 and AF167277, respectively. 


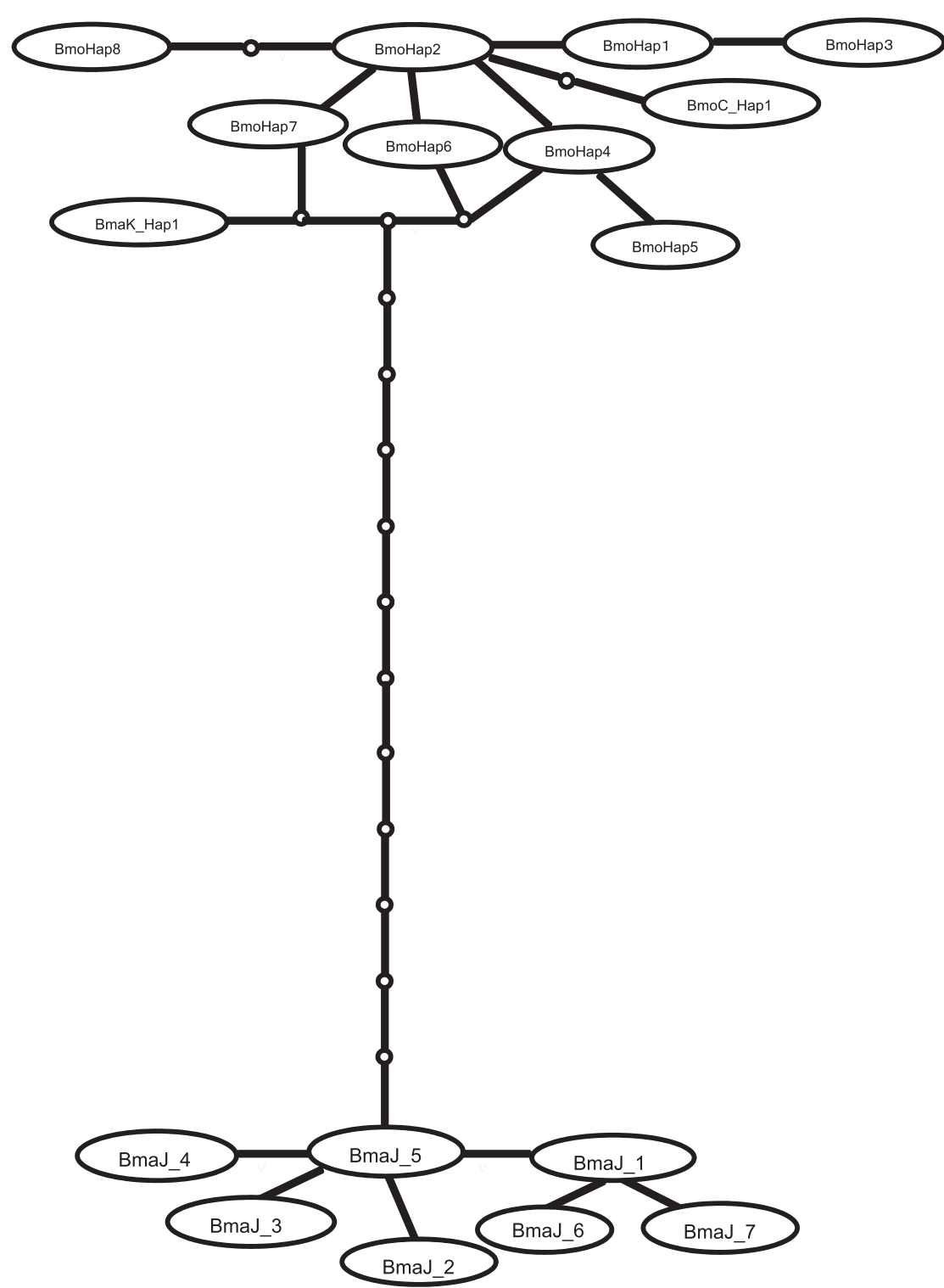

Fig. 4. Maximum-parsimony haplotype network of B. mori and Chinese, Korean, and Japanese B. mandarina COI coding sequences. A bar signifies a mutation. An open circle means an undetected haplotype.

gesting strict functional constraint on COI gene.

The noncoding region was excluded from this comparison because the differences in repetitive structure between $B$. mori and Japanese $B$. mandarina yielded unreliable nucleotide sequence alignment results. Nevertheless, we must emphasize the clear differences in the sequence composition of the NCDR as evidence for genetic differentiation between $B$. mori and Japanese $B$. mandarina (data not shown).

These observations support the lack of gene flow between $B$. mori and Japanese B. mandarina, despite the small number of comparisons. To thoroughly analyze this problem, a large-scale COI sequence analysis of Japanese B. mandarina populations is currently being performed.

This study was based on mitochondrial DNA analysis, and the possibility remains that the two species share a common nuclear gene haplotype. Taking genetic differentiation under artificial selection during domestication process into consideration, further studies on the nuclear genes will help to clarify the genetic relationship between $B$. mori and B. mandarina in Japan.

This work was supported by a grant from Fisheries of Japan the Ministry of Agriculture, Research project for genomics for Agricultural Innovation GAM-111.

We thank Nami Uwai and Naoko Kimura for providing technical support. We also thank Dr. Natuo Kômoto and Dr. Shuichiro Tomita for their valuable discussions. 


\section{REFERENCES}

Arunkumar, K. P., Metta, M., and Nagaraju, J. (2006) Molecular phylogeny of silkmoths reveals the origin of domesticated silkmoth, Bombyx mori from Chinese Bombyx mandarina and paternal inheritance of Antheraea proylei mitochondrial DNA. Mol. Phylogenet. Evol. 40, 419-427.

Astaurov, B. L., Garisheba, M. D., and Roginskaya, I. S. (1959) Chromosome complex of Ussuri geographical strain of Bombyx mandarina M. with special reference to the problem of the domesticated silkworm, Bombyx mori L. Cytology 1, 327-332.

Clement, M., Posada, D., and Crandall, K. A. (2000) TCS: a computer program to estimate gene genealogies. Mol. Ecol. 10, 1657-1659.

International Silkworm Genome Consortium (2008) The genome of lepidopteran model insect, the silkworm, Bombyx mori. Insect Biochem. Mol. Biol. 38, 1036-1045.

Kawaguchi, E. (1928) Zytologische Untersuchungen am Seidenspinner und seinen Verwandten. I. Gametogenese von Bombyx mori L. und Bombyx mandarina $\mathrm{M}$. und ihre Bastarde. Z. Zellforsch. Mikrosk. Anat. 7, 519-552.

Kim, K. Y., Lee, E. M., Lee, I. H., Hong, M. Y., Kang, P. D., Choi, K. H., Gui, Z. Z., Jin, B. R., Hwang, J. S., Ryu, K. S., et al. (2008) Intronic sequences of the silkworm strains of Bombyx mori (Lepidoptera: Bombycidae): High variability and potential for strain identification. Eur. J. Entomol. 105, $73-80$.

Kosegawa, E., Kikawada, T., Shimizu, K., and Okajima, T. (1997) Evaluation and preservation of silkworm genetic resources in Japan. In: Animal Genetic Resources: Efficient Conservation and Effective Use (ed.: T. Oishi), pp. 153-164. Research Council Secretarial of MAFF and National Institute of Agrobiological Resources, Tsukuba, Japan.

Kress, W. J., Wurdack, K. J., Zimmer, E. A., Weigt, L. A., and Janzen, D. H. (2005) Use of DNA barcodes to identify flowering plants. Proc. Natl. Acad. Sci. USA 102, 8369-8374.

Kumar, S., Nei, M., Dudley, J., and Tamura, K. (2008) MEGA: a biologist-centric software for evolutionary analysis of DNA and protein sequences. Brief. Bioinform. 9, 299-306.

Li, D., Guo, Y., Shao, H., Tellier, L. C., Wang, J., Xiang, Z., and Xia, Q. (2010) Characterization of mitochondrial genome of Chinese wild mulberry silkworm, Bombyx mandarina
(Lepidoptera: Bombycidae). BMC Evol. Biol. 10, 81.

Librado, P., and Rozas, J. (2009) DnaSP v5: software for comprehensive analysis of DNA polymorphism. Bioinformatics 25, 1451-1452.

Pan, M. H., Yu, Q. Y., Xia, Y. L., Dai, F. Y., Liu, Y. Q., Lu, C., Zhang, Z., and Xiang, Z. H. (2008) Characterization of mitochondrial genome of Chinese wild mulberry silkworm, Bomyx mandarina (Lepidoptera: Bombycidae). Sci. China Ser. C-Life Sci. 51, 693-701.

Sasaki, C. (1898) On the affinity of our wild and domestic silkworms. Ann. Zool. Jpn. 2, 33-40.

Simon, C., Frati, F., Beckenbach, A., Crespi, B., Liu, H., and Flook, P. (1994) Evolution, weighting and phylogenetic utility of mitochondria gene sequences and a compilation of conserved polymerase chain reaction primers. Ann. Entomic. Soc. Am. 87, 651-701.

Sorita, S. (1991) Preservation and utilization in genetic stocks of Bombyx mori. Natl. Inst. Seric. Entomol. Sci. Tsukuba (In Japanese).

Tamura, K., Dudley, J., Nei, M., and Kumar, S. (2007) MEGA4: Molecular Evolutionary Genetics Analysis (MEGA) software version 4.0. Mol. Biol. Evol. 24, 1596-1599.

Thompson, J. D., Higgins, D. G., and Gibson, T. J. (1997) CLUSTALW: improving the sensitivity of progressive multiple sequence alignment through sequence weighting, position-specific gap penalties and weight matrix choice. Nucleic Acids Res. 22, 4673-4680.

Tomita, S., Sohn, B. H., and Tamura, T. (1997) Cloning and characterization of a mariner-like element in the silkworm, Bombyx mori. Gen. Genet. Syst. 72, 219-228.

Xia, Q., Guo, Y., Zhang, Z., Li, D., Xuan, Z., Li, Z.-1., Dai, F., Li, Y., Cheng, D., Li, R., et al. (2009) Complete resequencing of 40 genomes reveals domestication events and genes in silkworm (Bombyx). Science 326, 433-436.

Yu, H. S., Shen, Y. H., Yuan, G. X. Hu, Y. G., Xu, H. E., Xiang, Z. H., and Zhang, Z. (2011) Evidence of selection at melanin synthesis pathway loci during silkworm domestication. Mol. Biol. Evol. 28, 1785-1799.

Yukuhiro, K., Sezutsu, H., Itoh, M., Shimizu, K., and Banno, Y. (2002) Significant levels of sequence divergence and gene rearrangements have occurred between the mitochondrial genomes of the wild mulberry silkmoth, Bombyx mandarina, and its close relative, the domesticated silkmoth, Bombyx mori. Mol. Biol. Evol. 19, 1385-1389. 